\title{
多维铁磁链型方程组*
}

周艈篥孙和生郭柏灵

(北京应角物理与计酋数学研究所, 非线性研究中心, 北京 100088)

摘要

本文考虑一类推广的多维铁磁链型方程组的齐次边值问题的整体可解性.

\section{关键词多维铁磁链型方程组、旋方程组、齐次边值问题、Galörkin 方法}

考虑铁磁链型推广组

$$
z_{s}={ }^{*}\left[g_{1}(z) \wedge g_{2}(z) \wedge \cdots \wedge g_{n-2}(z) \wedge \Delta z\right]+f(x, t, z),
$$

其中 $z=\left(z_{1}, z_{2}, \cdots, z_{n}\right)(n \geqslant 2)$ 是空间变量 $x=\left(x_{1}, \cdots, x_{m}\right) \in \mathbb{R}^{m}$ 和时间变量 $t \in \mathbb{R}^{+}$的 维未知矢量函数。符号“ $\wedge$ ”表示外乘算子, “ $\Delta$ ” 是 $\mathbb{R}^{m}(m \geqslant 1)$ 中的 Laplace 算子, “*” 表示 Hodge 星算子. 这里 $g_{k}(z)(k=1,2, \cdots, n-2)(n \geqslant 2)$ 是变量 $z \in \mathbb{R}^{*}$ 的 $n$ 维矢留 函数, $f(x, t, z)$ 是变量 $x \in \mathbb{R}^{*}, t \in \mathbb{R}^{+}, z \in \mathbb{R}^{n}$ 的 $n$ 维矢量函数.

$n=2$ 时方程组 (A) 变成非线性 Schrödinger 方程 ${ }^{[1-5]}$.

$n=3$ 和 $g_{1}(\boldsymbol{z}) \equiv \boldsymbol{z}$ 时这方程组成为熟知的铁磁链组 ${ }^{[6-13]}$.

方程组 (A) 是强耦合和强退化的 ${ }^{[2-5]}$.

在 $m=1$ 和 $g_{1}(z) \equiv z$ 的情形文㗘 [5] 考虑了方程组 (A) 的 Cauchy 问题; 在 $m=1$ 和 $f \equiv 0$ 的情形文献 [4]考虑了方程组 (A) 的几类边值问题.

本文在假定 $g_{1}(z) \equiv z, g_{k}(z)=a_{k}(k-2, \cdots, n-2), a_{k}$ 是 $\mathbb{R}^{n}$ 中的 $n$ 维常数矢量之 下,考虑多维情形 $m \geqslant 1$.

我们首先在柱形域 $Q_{T}=\left\{x \in \overline{\Omega \subset} \subset R^{m}, 0 \leqslant t \leqslant T\right\}$ 中考虑下面形式的推广的铁磁链型方 龧组:

$$
z_{0}={ }^{*}\left[z \wedge a_{2} \wedge \cdots \wedge a_{n-2} \wedge \Delta z\right]+f(x, t, z)
$$

的齐次边值问题, 其中 $z(x, t)=\left(z_{1}(x, t), \cdots, z_{n}(x, t)\right)$ 是 $n$ 维未知矢量函数, “ $\Delta$ ”是 $m$ 维 Euclid 空间 $\mathbb{R}^{m}$ 中的 Laplace 算子, $a_{k}(k=2, \cdots, n-2)$ 是 $\mathbb{R}^{n}$ 中 $n$ 维线性独立常数矢 量.

现在在 $Q_{T}$ 中对铁磁链型方程组 (1) 考虑齐次边值问题 ${ }^{[12]}$

$$
\boldsymbol{z}(x, t)=0, x \in \partial 0 ; 0 \leqslant t \leqslant T
$$

和初值条件

- 国家目然科学基金和中国工程物理研究院落全资助项目. 


$$
z(x, l)=\varphi(x), x \in \bar{\Omega},
$$

其中 $\varphi(x)=\left(\varphi_{1}(x), \cdots, \varphi_{n}(x)\right)$ 是 $\bar{\Omega}$ 中已给的 $n$ 维初始矢是函数. 我们对强裸含和强 退化组(1)的齐次边值问题 (2),(3) 建立解解且对对应的旋方程组

$$
z_{1}=\varepsilon \Delta z+{ }^{*}\left[z \wedge a_{2} \wedge \cdots \wedge a_{n-2} \wedge \Delta z\right]+f(x, t, z)
$$

亦考县边值问题 (2),(3).

我们利用 Galërkin 方法 ${ }^{[12]}$ 构造上述问题的近似解。令 $\omega_{n}(x)(n=1,2, \cdots)$ 是具有 齐次边界条件 $\left.u\right|_{\partial Q}=0$ 的汀程 $\Delta u+\lambda u=0$ 在两次连续可微的有界域 $Q$ 中对应于本征值 $2_{n}(n=1,2, \cdots)$ 的正规化本征函数. 于是 $\left\{\omega_{n}(x)\right\}$ 组成本征函数的正规化正交系.

令旋转组(4)的齐次边值问题 (2), (3) 何近似解 $z^{N}(x, t)$ 長为

$$
z^{N}(x, t)=\sum_{n=1}^{N} \alpha_{n N}(t) \omega_{n}(x),
$$

其中 $\alpha_{n N}(t)(n-1,2, \cdots, N ; N=1,2, \cdots)$ 是变量 $t \in \mathbb{R}^{+}$的未知系数矢昌函数。根据 Galërkin 方法, 系数矢量 $\alpha_{n N}(b)(n=1,2, \cdots, N ; N=1,2, \cdots)$ 被假定满足下列一阶常涢 分方程组:

$$
\begin{aligned}
& \int_{0} z_{s}^{N}(x, t) \omega_{s}(x) \mathrm{d} x=\varepsilon \int_{0} \omega_{s}(x) \Delta z^{N}(x, t) \mathrm{d} x \\
& \left.\quad+\int_{0} \omega_{s}(x)^{*} \mathrm{i} z^{N}(x, t) \wedge a_{2} \wedge \cdots \wedge a_{n-2} \wedge \Delta z^{N}(x, t)\right] \mathrm{d} x \\
& \quad+\int_{0} \omega_{s}(x) f\left(x, t, z^{N}(x, t)\right) \mathrm{d} x, s=1,2, \cdots, N
\end{aligned}
$$

和初始条件

$$
\alpha_{s N}(0)=\int_{Q} \omega_{s}(x) r(x) \mathrm{d} x, s=1,2, \cdots, N .
$$

假设方程组(1)和(4)以及初始条件(3)满足下列条件:

(I) $n \times n$ Jacobi 微商矩阵 $\partial f(x, t, z) / \partial z$ 是半有界的, 即, 存在常数 $b$ 使得

$$
\xi \cdot \frac{\partial f(x, t, z)}{\partial z} \xi \leqslant b|\xi|^{2}
$$

其中“.”表示 $\boldsymbol{n}$ 维 Euclid 空问中的标晴乘;

(II) 方程组 (1) 积(4) 是齐次的, 即 $f(x, t, 0) \equiv 0$. 且假定有

$$
|f(x, t, z)| \leqslant A|z|^{t}+B,|\nabla f(x, t, z)| \leqslant A|z|^{1+\frac{2}{m}}+B,
$$

其中 $2 \leqslant l<2+\frac{4}{m-2}(m \geqslant 2) ; A$ 和 $B$ 是非负常数且 $\nabla$ 表示关于变星 $x \in \mathbb{R}^{n}$ 的编 度算子；

(III) $n$ 维矢量函娄 $\varphi(x) \in H_{0}^{\prime}(\Omega)$.

现在来给出 Galërkin 方法的近似解 $z^{N}(x, t)$ 的一些估计.

引理 1 假定 Jacobi 微商矩阵 $\partial f / \partial z$ 是半有界的且 $\varphi \in L_{2}(\Omega)$. 对近似解 $z^{N}(x, l)$ $(N=1,2, \cdots)$ 有估计

$$
\sup _{0 \leqslant s \leqslant T} \| z^{N}(\cdot, t)_{L_{2}(\theta)} \leqslant K_{1},
$$

其中常数 $K_{1}$ 不依赖于 $\varepsilon>0$ 和 $N$ 。 


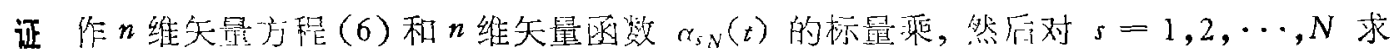
和, 我们得等式

$$
\begin{aligned}
\int_{0} z_{z}^{\mathrm{V}} \cdot z^{N}=\varepsilon & \int_{\Omega} \Delta z^{N} \cdot z^{N}+\int_{\Omega} z^{N} \cdot{ }^{*}\left[z^{N} \wedge a_{2} \wedge \cdots \wedge a_{n-2} \wedge \Delta z^{N}\right] \\
& +\int_{g} z^{N} \cdot f\left(x, t, z^{N}\right),
\end{aligned}
$$

这里我们有

$$
\int_{\Omega} z_{s}^{N} \cdot z^{N}=\frac{1}{2}-\frac{\mathrm{d}}{\mathrm{d} t}\left\|z^{N}(\cdot, t)\right\|_{L_{2}(Q)} \text { 利 } \int_{\Omega} z^{N} \cdot{ }^{*}\left[z \wedge a_{2} \wedge \cdots \wedge a_{n-2} \wedge \Delta z^{N}\right]=0 。
$$

对(11)式在端第一个积分存

$$
\int_{\Omega} \Delta z^{N} \cdot z^{N}=\int_{\partial Q}\left(\nabla z^{N} \cdot z^{N}\right) \circ \gamma_{m}-\int_{\Omega} \nabla z^{N} \nabla z^{N},
$$

其中 $\gamma_{m}$ 是 $\partial Q$ 上的单位法间知量， $\nabla z^{N}$ 是 $m \times n$ 矩阵或张量，“。”表示 $m$ 维 Euclid 空间 $x \in R^{m}$ 中的标量器。由齐次边界条件(2)我们有

$$
\int_{s} \Delta z^{N} \cdot z^{N}=-\left\|\nabla z^{N}(\cdot, t)\right\|_{L_{2}(\theta)}^{z}
$$

对 (11) 式韨盾的积分牫们有

$$
\begin{aligned}
\int_{0} f\left(x, t, z^{N}\right) \cdot z^{N} & =\int_{0} z^{N} \cdot\left[\int_{0}^{1} \frac{\partial f\left(x, \imath, \tau z^{N}\right)}{\partial z} \cdot z^{N} \mathrm{~d} \tau+f(x, t, 0)\right], \\
& \leqslant\left(b+\frac{1}{2}\right)\left\|z^{N}(\cdot, t)\right\|_{L_{2}(\Omega)}^{z}+\frac{1}{2}\|f(\cdot, t, 0)\|_{L_{2}(\Omega)}^{2},
\end{aligned}
$$

于是(11) 式脑为

由此引理得证。

$$
\begin{aligned}
& \frac{\mathrm{d}}{\mathrm{d} t}\left\|z^{N}(\cdot, t)\right\|_{L_{z}(\theta)}^{2}+2 \varepsilon\left\|\nabla z^{N}(\cdot, t)\right\|_{L_{2}(\theta)}^{2} \\
& \quad \leqslant(2 b+1)\left\|z^{N}(\cdot, t)\right\|_{L_{2}(\theta)}^{2}+\|f(\cdot, t, 0)\|_{L_{2}(\theta)}^{2} .
\end{aligned}
$$

引理 2 在假定 (I), (II) 和 (III) 之下, 对近似慨 $z^{N}(x, t)$ 有估计

$$
\sup _{0 \leqslant s \leqslant T}\left\|\nabla z^{N}(\cdot, t)\right\|_{\boldsymbol{L}_{2}(\Omega)}+\sqrt{\varepsilon}\left\|\Delta z^{N}(\cdot, t)\right\|_{\left.L_{2}(S)\right)} \leqslant K_{2},
$$

其中常数 $K_{2}$ 不依制于 $\varepsilon>0$ 和 $N$.

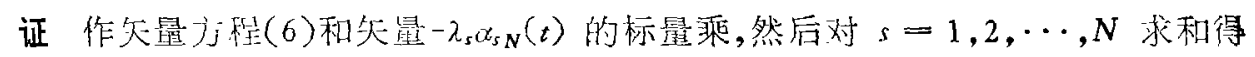

$$
\begin{aligned}
\int_{0} z_{1}^{N} \cdot \Delta z^{N}=\varepsilon & \int_{\Omega} \Delta z^{N} \cdot \Delta z^{N}+\int_{\Omega} \Delta z^{N} \cdot{ }^{*}\left[z^{N} \wedge a_{2} \wedge \cdots \wedge a_{n-2} \wedge \Delta z^{N}\right] \\
& +\int_{\Omega} \Delta z^{N} \cdot f\left(x, t, z^{N}\right) .
\end{aligned}
$$

上面等式帅的第一个积分可以表为

$$
\int_{S} z_{i}^{N} \cdot \Delta z^{N}=\int_{\partial Q}\left(z_{i}^{N} \cdot \nabla z^{N}\right) \circ \gamma_{m}-\int_{Q} \nabla z_{i}^{N}: \nabla z^{N}=-\frac{1}{2} \frac{\mathrm{d}}{\mathrm{d} t}\left\|\nabla z_{t}^{N}(\cdot, t)\right\|_{L_{2}(a)}^{2} .
$$

对最后的积多有

$$
\int_{0} f\left(x, t, z^{N}\right) \cdot \Delta z^{N}=\int_{\partial Q}\left(f\left(x, t, z^{N}\right) \cdot \nabla z^{N}\right) \circ \gamma_{z}-\int_{Q} \operatorname{Df}\left(x, t, z^{N}\right) \circ \nabla z^{N},
$$


其中“ $D ”$ 表示全梯度算子, 而“ $\nabla$ ”则表示关于变是 $x \in \mathbb{R}^{m}$ 的傓梯度算子。这里

$$
\operatorname{D} f\left(x, t, z^{N}\right)=\nabla f\left(x, t, z^{N}\right)+\frac{\partial f\left(x, t, z^{N}\right)}{\partial z} \cdot \nabla z^{N},
$$

因为方程组 (4) 是齐次的,于是我们有

$$
\int_{0} f\left(x, t, z^{N}\right) \cdot \Delta z^{N}=-\int_{0} \nabla f\left(x, t, z^{N}\right): \nabla z^{N}-\int_{Q}\left(\frac{\partial f\left(x, t, z^{N}\right)}{\partial z} \cdot \nabla z^{v}\right) \circ \nabla z^{N} \cdot
$$

由于 $\partial f\left(x, t, z^{N}\right) / \partial z$ 是半有界的, 有

$$
\int_{0}\left(\frac{\partial f\left(x, t, z^{N}\right)}{\partial z} \cdot \nabla z^{N}\right) \circ \nabla z^{N} \leqslant b\left\|\nabla z^{N}(\cdot, t)\right\|_{L_{2}(\Omega)}^{2}
$$

另外，

$$
\left|\int_{\Omega} \nabla f\left(x, t, z^{N}\right): \nabla z^{N}\right| \leqslant \frac{1}{2}\left\|\nabla z^{N}(\cdot, t)\right\|_{L_{2}(\Omega)}^{2}+\frac{1}{2}\left\|\nabla f\left(\cdot, t, z^{N}(\cdot, t)\right)\right\|_{L_{2}(\rho)}^{2} \bullet
$$

利用矢量函数 $f(x, t, z)$ 的性质 $(9)$, 有

$$
\|\nabla f(\cdot, t, z(\cdot, t))\|_{L_{2}(0)}^{2} \leqslant C_{1} \int_{Q}\left|z^{N}(x, t)\right|^{2+\frac{4}{m}} \mathrm{~d} x+C_{2},
$$

其中 $C_{1}$ 和 $C_{2}$ 是常数. 利用对 Sobolev 空间的插值公式, 可以验证得

$$
\int_{\Omega}\left|z^{N}(x, t)\right|^{2+\frac{4}{m}} \mathrm{~d} x \leqslant C_{3}\left\|z^{N}(\cdot, t)\right\|_{L_{2}(\Omega)}^{\frac{4}{m}}\left\|\nabla z^{N}(\cdot, t)\right\| L_{L_{2}(\theta)}+C_{4}\left\|z^{N}(\cdot, t)\right\|^{2+\frac{4}{m}}{ }_{L_{2}(\theta)} \text {. }
$$

其中 $C_{3}$ 和 $C_{4}$ 是常数。

最后, (13) 式成为

$$
\begin{aligned}
-\frac{\mathrm{d}}{\mathrm{d} t} \| & \nabla z^{N}(\cdot, t)\left\|_{i_{2}(\Omega)}^{2}+2 \varepsilon\right\| \Delta z^{N}(\cdot, t) \|_{L_{2}(\Omega)}^{2} \\
& \leqslant\left(2 b+C_{5}\right)\left\|\nabla z^{N}(\cdot, t)\right\|_{L_{2}(\Omega)}^{2}+C_{6},
\end{aligned}
$$

其中 $C_{5}$ 和 $C_{6}$ 为常数, 依赖于 $\sup _{0 \leqslant s \leqslant T}\left\|z^{N}(\cdot, t)\right\|_{L_{2}(\theta)}$ 但不依赖于 $\varepsilon>0$ 和 $N$.

直接利用 Sobolev 空问的揷犆公式作为推论我们有下面引留。

引理 3 在条件 (I), (II) 和 (III) z下, 对近似解 $z^{N}(x, t)$ 有估计

$$
\sup _{0 \leqslant s \leqslant r}\left\|z^{N}(\cdot, t)\right\|_{L_{l}(Q)} \leqslant K_{3},
$$

其中 $2 \leqslant l<\frac{2 m}{m-2}, K_{3}$ 是不依赖于 $\varepsilon>0$ 和 $N$ 的常数.

引理 4 假设条件 (I), (II) 和 (III) 满足. 近似解 $z^{N}(x, t)$ 有

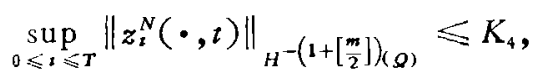

其中 $K_{4}$ 是不依赖于 $\varepsilon>0$ 和 $N$ 的拧数。

证 令 $v(x) \in H_{0}^{1+\left[\frac{m}{2}\right]}(Q)$ 是任一试验函数。有

$$
v(x)=v^{N}(x)+\bar{v}^{N}(x),
$$

其中 $v^{N}(x)=\sum_{n=1}^{N} \beta_{n} \omega_{n}(x), \bar{v}^{N}(x)=\sum_{n=N+1}^{\infty} \beta_{n} \omega_{n}(x)$ 且 $\beta_{n}=\int_{0} v(x) \omega_{n}(x) \mathrm{d} x, n-1,2$, 
对 $s \geqslant N+1$ 我们有 $\int_{\Omega} z_{s}^{N} \omega_{s}=0$.

对 $1 \leqslant s \leqslant N$ 有

$$
\begin{aligned}
\int_{0} z_{i}^{N} \omega_{s}= & \int_{\partial \Omega} \omega_{s}\left(\gamma_{m} \circ^{*}\left[z^{N} \wedge a_{2} \wedge \cdots \wedge a_{n-2} \wedge \nabla z^{N}\right]\right) \\
& -\int_{\Omega} \nabla \omega_{s} \circ^{*}\left[z^{N} \wedge a_{2} \wedge \cdots \wedge a_{n-2} \wedge \nabla z^{N}\right] \\
& +\varepsilon \int_{\partial Q} \omega_{s}\left(\nabla z^{N} \circ \gamma_{m}\right)-\varepsilon \int_{\Omega} \nabla \omega_{s} \circ \nabla z^{N} \\
& +\int_{\Omega} \omega_{s} f\left(x, t, z^{N}\right) .
\end{aligned}
$$

(16)式右端的第一个和第三个积分等于零。对于第二个积分有

$$
\begin{aligned}
& \mid \int_{\Omega} \nabla \omega_{s} 0^{*}\left[z^{N} \wedge a_{2} \wedge \cdots \wedge a_{n-2} \wedge \nabla z^{N}\right] \\
& \quad \leqslant C_{7}\left\|\nabla \omega_{s}\right\|_{L_{p}(\Omega)}\left\|z^{N}(\cdot, t)\right\|_{L_{l}(\Omega)}\left\|\nabla z^{N}(\cdot, t)\right\|_{L_{2}(\Omega)},
\end{aligned}
$$

其中 $\frac{1}{p}+\frac{1}{l}+\frac{1}{2}=1$ 且 $2 \leqslant l<\frac{2 m}{m-2}$. 于是我们有 $p>m$. 由 Sobolev 空间的嵌人 定理,我们有

$$
\left\|\nabla \omega_{s}\right\|_{L_{p}(\Omega)} \leqslant C_{8}\left\|\omega_{s}\right\|_{\left.H^{1+\left[\frac{m}{2}\right.}\right]_{(\Omega)}},
$$

其中 $P=m+\delta$ 且 $\delta>0$ 小. 因此

$$
\mid \int_{0} \nabla \cdot \omega_{s} \cdot{ }^{*}\left[z^{N} \wedge a_{2} \wedge \cdots \wedge a_{n-2} \wedge \nabla z^{N}\right] \leqslant C_{9}\left\|\omega_{s}\right\|_{\left.H^{1+\left[\frac{m}{2}\right.}\right]_{(0)}},
$$

其中 $C$, 是依赖于 $\sup _{0 \leqslant s}\left\|z^{N}(\cdot, t)\right\|_{L_{2}(\varrho)}$ 的常数, 但不依赖于 $0 \leqslant t \leqslant T$ 和 $N_{\circ}$

对第四个积分有

$$
\left|\int_{\Omega} \nabla z^{N} \circ \nabla \omega_{s}\right| \leqslant C_{10}\left\|\nabla z^{N}(\cdot, t)\right\|_{L_{2}(\Omega)}\left\|\nabla \omega_{s}\right\|_{L_{2}(\Omega) \bullet}
$$

对(16)式中最后的积分由条件（II）盾

$$
\begin{aligned}
\left|\int_{Q} \omega_{s} f\left(x, t, z^{N}\right)\right| & \leqslant\left\|\omega_{s}\right\|_{L_{\infty}(\Omega)} \int_{Q}\left|f\left(x, t, z^{N}\right)\right| \\
& \leqslant\left\|\omega_{s}\right\|_{L_{\infty}(\Omega)}\left\{A \int_{\Omega}\left|z^{N}(x, t)\right|^{t} \mathrm{~d} x+B\right\} \\
& \leqslant C_{11}\left\|\omega_{s}\right\|_{L_{\infty}(S)} \leqslant C_{12}\left\|\omega_{s}\right\|_{\left.H^{1+\left[\frac{m}{2}\right.}\right]_{(\Omega)}},
\end{aligned}
$$

其中 $\int_{\varrho}\left|z^{N}(\cdot, t)\right|^{l} \leqslant C_{13}\left\|z^{N}(\cdot, t)\right\|\left\|_{L_{2}(0)}^{(1-a) t}\right\| z^{N}(\cdot, t)\|\|_{I^{\prime}(0)}^{a l}$

且 $\alpha=\frac{m}{2}-\frac{m}{l} \leqslant 1$ 当 $2 \leqslant l<\frac{2 m}{m-2}$. 因此最后得

$$
\left|\int_{\Omega} \omega_{s} z_{t}^{N}\right| \leqslant C_{1+}\left\|\omega_{s}\right\|_{\left.H^{1+\left[\frac{m}{2}\right.}\right]_{(\Omega)}},
$$

其中 $C_{14}$ 是依赖于 $\sup _{0 \leqslant t \leqslant T}\left\|z^{N}(\cdot, t)\right\|_{L_{2}(\Omega)}$ 的常数, 但不依赖于 $\varepsilon>0,0 \leqslant t \leqslant T$ 和 $N$. 于是我们有 


$$
\left|\int_{0} z_{\imath}^{N} v^{N}\right| \leqslant C_{14}\left\|v^{N}\right\|_{H^{1}+\left[\begin{array}{l}
m \\
2
\end{array}\right]_{(0)}}
$$

且最后有

$$
\left|\int_{Q} z_{i}^{N} v\right| \leqslant C_{1+}\|v\|_{H^{1}+\left[\frac{m}{2}\right]_{(\Omega)}}, \forall v \in H_{0}^{1+\left[\frac{m}{2}\right]}(\Omega), \quad 0 \leqslant t \leqslant T .
$$

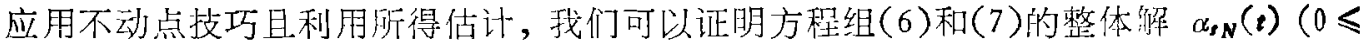
$t \leqslant T ; s=1,2, \cdots, N)$ 的存在性.

引理 5 假设条件 (I), (II) 和 (III) 满足。对常微分方程组(6)的初值问题(7)有至少 一个整体连续可溦解 $\alpha_{s N}(t)(0 \leqslant t \leqslant T ; s=1,2, \cdots, N)$.

引理 6 在条件 (I), (II) 和 (III) 已下, 对近似解 $z^{N}(x, t)$ 有

$$
\left\|z^{N}(\cdot, t+\Delta t)-z^{N}(\cdot, t)\right\|_{L_{2}(\Omega)} \leqslant K_{5} \Delta t^{\frac{1}{2+\left[\frac{m}{2}\right]}},
$$

其中 $K_{5}$ 不依赖于 $0 \leqslant t \leqslant T, t+\Delta t \leqslant T, \varepsilon>0$ 和 $N$.

证 从 Hilbert 空间具有负指标的插值公式得

$$
\left\|z^{N}(\cdot, t)\right\|_{L_{2}(\Omega)} \leqslant C_{15}\left\|z^{N}(\cdot, t)\right\|_{H^{-}\left(1+\left[\begin{array}{c}
m \\
2
\end{array}\right]\right)(\Omega)}^{\frac{1}{2+\left[\begin{array}{l}
m \\
2
\end{array}\right]}}\left\|z^{N}(\cdot, t)\right\|_{H^{1}(\Omega)}^{\frac{1+\left[\begin{array}{c}
m \\
2
\end{array}\right]}{2+\left[\begin{array}{l}
m \\
2
\end{array}\right]}}, \forall 0 \leqslant t \leqslant T .
$$

将此公式应用到 $n$ 维矢最函数 $z^{N}(x, t+\Delta t)-z^{N}(x, t)$ 上得到

$$
\begin{gathered}
\left\|z^{N}(\cdot, t+\Delta t)-z^{N}(\cdot, t)\right\|_{L_{t}(Q)} \\
\leqslant C_{15}\left\|z^{N}(\cdot, t+\Delta t)-z^{N}(\cdot, t)\right\|_{\left.\left.H^{-\left(1+\left[\frac{m}{2}\right]\right.}\right]\right)_{(\Omega)}}^{\frac{1}{2+\left[\frac{m}{2}\right]}}\left\|z^{N}(\cdot, t+\Delta t)-z^{N}(\cdot, t)\right\|_{H^{\prime}(\Omega)}^{\frac{1+\left[\frac{m}{2}\right]}{2+\left[\frac{m}{2}\right]}},
\end{gathered}
$$

其中 $0 \leqslant t, t+\Delta t \leqslant T$. 于是

$$
\begin{aligned}
& \left\|z^{N}(\cdot, t+\Delta t)-z^{N}(\cdot, t)\right\|_{L_{2}(\theta)}
\end{aligned}
$$

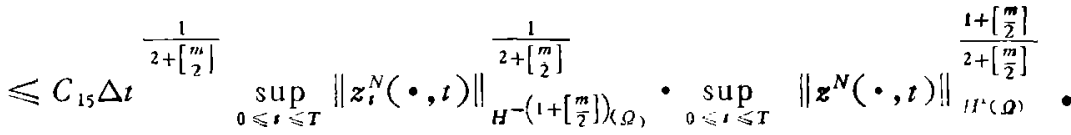

这就证明了引理。

为了要得到铁磁链型的非退化抛物的旋方程组 (4)和退化方程组(1)的齐次边值问题(2),

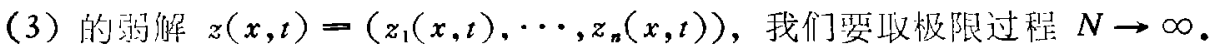

定义 $1 n$ 维矢最函数 $z(x, t)=\left(z_{1}(x, t), \cdots, z_{n}(x, t)\right) \in L_{0}\left(0, T ; H_{1}^{\prime}(\Omega)\right)$ 是铁磁链 型掞方程组 (4) $(\varepsilon>0)$ 或方程组 (1) $(\varepsilon=0)$ 的齐次边值问题 (2), (3) 的弱解, 如果对任意 的试验函数 $\phi(x, t) \in \mathrm{C}^{(1)}\left(Q_{T}\right), \psi(x, t)=0$ 在 $\{x \in \Omega, t=T\} \cup\{x \in \partial \Omega, 0 \leqslant t \leqslant T\}$ 上, 成立积分关系式

$$
\begin{aligned}
& \iint_{Q_{T}}\left\{\psi_{s}(x, t) z(x, t)-\nabla \phi(x, t) \circ^{*}\left[z(x, t) \wedge a_{2} \wedge \cdots \wedge a_{n-2} \wedge \nabla z(x, t)\right]\right. \\
& \quad-\varepsilon \nabla \phi(x, t) \circ \nabla z(x, t)+\phi(x, t) f(x, t, z(x, t))\} \mathrm{d} x \mathrm{~d} t \\
& \quad+\int_{\Omega} \psi(x, 0) \phi(x) \mathrm{d} x=0,
\end{aligned}
$$


其中 $\varepsilon \geqslant 0$; 而且 $z(x, t)$ 在柱形域 $Q_{T}$ 的侧面流形 $\partial Q \times[0, T]$ 上几乎处处满足济次边 蒀条件 $(2)$.

对任意的试验函数 $\phi(x, t)$, 有对应的 Galërkin 近似

$$
\psi^{N}(x, t)=\sum_{n=1}^{N} \beta_{n N}(t) \omega_{n}(x)
$$

在 $\mathrm{C}^{(1)}\left(Q_{T}\right)$ 中当 $N \rightarrow \infty$ 时一致收敛于 $\phi(x, t)$, 其中 $\beta_{s s}(t)=\int_{\Omega} \phi(x, t) \omega_{s}(x) \mathrm{d} x(s=$ $1,2, \cdots, N)$. 由方程组 (6) 立即导出积分关系式

$$
\begin{aligned}
& \iint_{Q_{T}}\left\{\phi_{1}^{N}(x, t) z^{N}(x, t)-\nabla \phi^{N}(x, t) \circ^{*}\left[z^{N}(x, t) \wedge a_{2} \wedge \cdots \wedge a_{n-2} \wedge \nabla z^{N}(x, t)\right]\right. \\
& \left.\quad-\varepsilon \nabla \psi^{N}(x, t) \circ \nabla z^{N}(x, t)+\psi^{N}(x, t) f\left(x, t, z^{N}(x, t)\right)\right\} \mathrm{d} x \mathrm{~d} t \\
& \quad+\int_{\delta} \phi^{N}(x, 0) \varphi^{N}(x) \mathrm{d} x=0 .
\end{aligned}
$$

从前面所得近似解 $z^{N}(x, t)$ 的先验估计, 我们有: $\left\{z^{N}(x, t)\right\}$ 在泛函数空间

$$
G \equiv L_{\infty}\left(0, T ; H_{0}^{\prime}(\Omega)\right) \cap W_{\infty}^{(1)}\left(0, T ; H^{-\left(1+\left[\begin{array}{c}
m \\
2
\end{array}\right]\right)}(\Omega)\right)
$$

中关于 $N$ 是一致有界们。

因此，从近似解的序列 $\left\{z^{N}(x, t)\right\}$ 的我们可以选取一子序列 $\left\{z^{N_{i}}(x, t)\right\}$, 且还有一 $n$ 维矢量函数 $z(x, t) \in L_{p}\left(0, T ; H^{1}(\Omega)\right)(1<p<\infty)$, 使得 $\left\{z^{N_{1}}(x, t)\right\}$ 在 $L_{\rho}\left(0, T ; L_{2}(\Omega)\right)$ 中收敛于 $z(x, t)$, 且 $\left\{\nabla z^{N_{i}}(x, t)\right\}$ 在 $L_{p}\left(0, T ; L_{2}(Q)\right)$ 中弱收敛于对应的微商 $\nabla z(x, t)$.

另一方面, $\left\{z_{i}^{N}(x, t)\right\}$ 在: $L_{p}\left(0, T ; H^{-\left(1+\left[\frac{m}{2}\right]\right)}(Q)\right)(1<p<\infty)$ 中一致存界, 因此它弱 收敛于一 $n$ 维矢量函数 $w(x, t) \in L_{p}\left(0, T ; H^{-\left(1+\left[\frac{m}{2}\right]\right)}(\Omega)\right)(1<p<\infty)$. 我们考虑积分恒 等式

$$
\iint_{Q} z_{T}^{N} \cdot(x, t) \psi(x, t) \mathrm{d} x \mathrm{~d} t=-\iint_{Q_{T}} z^{N_{i}}(x, t) \psi_{,}(x, t) \mathrm{d} x \mathrm{~d} t,
$$

其中 $\phi(x, t)$ 是在开柱形域 $\{x \in \Omega ; 0<t<T\}$ 中具有有限支柱的试验函数. 取极限 $N_{i} \rightarrow$ 我们得

$$
\int_{Q_{T}} w(x, t) \phi(x, t) \mathrm{d} x \mathrm{~d} t=-\iint_{Q_{T}} z(x, t) \psi_{t}(x, t) \mathrm{d} x \mathrm{~d} t .
$$

这表明 $w(x, t)=z_{\imath}(x, t)$, 图此 $\left\{z_{r}^{N}(x, t)\right\}$ 在 $L_{p}\left(0, T ; H^{-\left(1+\left[\begin{array}{l}m \\ 2\end{array}\right]\right)}(\Omega)\right)$ 中弱收玫于 $z_{s}(x, t)$. 这样 $n$ 维板限尔是函数 $z(x, t)$ 鹤于

$$
G_{p} \equiv L_{p}\left(0, T ; H^{1}(\Omega)\right) \cap W_{p}^{(1)}\left(0, T ; H^{-\left(1+\left[\frac{m}{2}\right]\right)}(\Omega)\right), \quad 1<p<\infty .
$$

由于 $z(x, t)$ 在 $G_{p}$ 中的范数炎于 $1<p<\infty$ 是一致有界的，因此 $z(x, t)$ 属于泛函空间 $G \equiv G_{\infty}$.

这样我们得到下面的引理。

引理 7 假设条作 (I), (II) 和 (III) 满足. 近似解序列 $\left\{z^{N}(x, t)\right\}$ 的 $n$ 维极限矢量函 
数 $z^{\varepsilon}(x, t) \in G(\varepsilon \geqslant 0)$ 有估计

$$
\begin{gathered}
\sup _{0 \leqslant s \leqslant T}\left\|z^{\varepsilon}(\cdot, t)\right\|_{H^{1}(\Omega)}+\sup _{0 \leqslant t \leqslant T}\left\|z_{s}^{\varepsilon}(\cdot, t)\right\|_{H^{-}\left(1+\left[\begin{array}{l}
m \\
2
\end{array}\right]\right)_{(\Omega)}} \leqslant K_{6}, \\
\left\|z^{s}(\cdot, t+\Delta t)-z^{\varepsilon}(\cdot, t)\right\|_{L_{1}(\Omega)} \leqslant K_{7} \Delta t^{\frac{1}{2+\left[\frac{m}{2}\right]}},
\end{gathered}
$$

其中 $K_{6}$ 和 $K_{7}$ 是不依赖于 $0 \leqslant t, t+\Delta t \leqslant T$ 和 $\varepsilon \geqslant 0$ 的常数.

从序列 $\left\{z^{N_{i}}(x, t)\right\}$ 我们可以再选取一子序列 $\left\{z^{N_{i}^{*}}(x, t)\right\}$, 使得 $\left\{z^{N^{\prime}}(x, t),\right\}$, 在 $L_{2}(\Omega)$ 中对所有 $0 \leqslant t \leqslant T$ 强收玫于 $z(x, t)$, 且 $\left\|z^{N_{i}^{\prime}}(\cdot, t)\right\|_{L_{2}(\Omega)}$ 当 $N_{i}^{\prime} \rightarrow \infty$ 时关于 $0 \leqslant t \leqslant T$

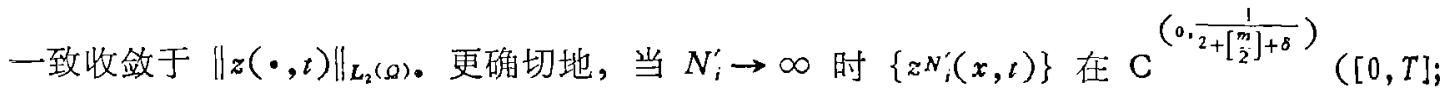
$\left.L_{2}(Q)\right)$ 中对 $\delta>0$ 收敛于 $z(x, t)$.

因此 $z(x, t)$ 在柱形域 $Q_{T}$ 的侧面流形 $\partial Q \times[0, T]$ 上几乎处处满足齐次边界条件 (2).

现在考虑对 $N_{i}^{\prime} \rightarrow \infty$ 积分关系式 (19) 的极限过程. 当 $N_{i}^{\prime} \rightarrow \infty$ 时 $\left\{\psi_{i}^{N_{i}^{\prime}}(x, t)\right\}$ 和 $\left\{\nabla \psi^{\prime},(x, t)\right\}$ 在柱形域 $Q_{T}$ 中分别一致收敛于 $\psi_{i}(x, t)$ 和 $\nabla \psi(x, t) ;\left\{\psi_{i}^{\prime}(x, 0)\right\}$ 在 $O$ 中一 致收玫于 $\phi(x, 0)$, 且 $\left\{\varphi^{N^{\prime}}(x)\right\}$ 在 $L_{2}(\Omega)$ 中收敛于 $\varphi(x)$.

对 (19)式中第一个积分的被积函数的第二部分有

$$
\begin{aligned}
& \left|\iint_{Q_{T}} \nabla \psi^{N \circ}{ }^{*}\left[z^{N} \wedge a_{2} \wedge \cdots \wedge a_{n-2} \wedge \nabla z^{N}\right]-\iint_{Q_{T}} \nabla \psi_{0} 0^{*}\left[z \wedge a_{2} \wedge \cdots \wedge a_{n-2} \wedge \nabla z\right]\right| \\
& \leqslant\left|\iint_{Q_{T}}\left(\nabla \psi^{N}-\nabla \psi\right) 0^{*}\left[z^{N} \wedge a_{2} \wedge \cdots \wedge a_{n-2} \wedge \nabla z^{N}\right]\right| \\
& +\left|\iint_{Q_{T}} \nabla \psi \circ^{*}\left[\left(z^{N}-z\right) \wedge a_{2} \wedge \cdots \wedge a_{n-2} \wedge \nabla z^{N}\right]\right| \\
& +\iiint_{Q_{T}^{\prime}} \nabla \phi \nu^{*}\left[z \wedge a_{2} \wedge \cdots \wedge a_{n-2} \wedge\left(\nabla z^{N}-\nabla z\right)\right] \\
& \leqslant\left\|\nabla \psi^{N}-\nabla \psi\right\|_{L_{\infty}\left(Q_{T}\right)}\left\|z^{N}\right\|_{L_{2}\left(Q_{T}\right)}\left\|\nabla z^{N}\right\|_{L_{2}\left(Q_{T}\right)} \\
& +\|\nabla \psi\|_{L_{\infty}\left(Q_{T}\right)}\left\|z^{N}-z\right\|_{L_{L}\left(Q_{T}\right)}\left\|\nabla z^{N}\right\|_{L_{2}\left(Q_{T}\right)} \\
& +\|\nabla \phi\|_{L_{\infty}\left(Q_{T}\right)}\|z\|_{L_{2}\left(Q_{T}\right)}\left\|\nabla z^{N}-\nabla z\right\|_{L_{2}\left(Q_{T}\right)} \text {. }
\end{aligned}
$$

当 $N_{i}^{\prime} \rightarrow \infty$ 时上面不等式右端的所有项都趋于零.

因为 $\left\{z^{N^{\prime}},(x, t)\right\}$ 强收敛于 $z(x, t)$, 于是 $\left\{z^{N_{i}^{\prime}}(x, t)\right\}$ 在 $Q_{T}$ 中几乎处处收敛于 $z(x, t)$, 且 $\left\{f\left(x, t, z^{N^{\prime}},(x, t)\right)\right\}$ 在 $Q_{T}$ 中亦几乎处处收玫于 $f(x, t, z(x, t))$. 对任意 $0 \leqslant t \leqslant T$, 从象 件 (9) 我们得

$$
\begin{aligned}
\left\|f\left(\cdot, t, z^{N}(\cdot, t)\right)\right\|_{L_{q}(o)} & =\int_{\Omega}\left|f\left(x, t, z^{N}(x, t)\right)\right|^{q} \mathrm{~d} x \\
& \leqslant C_{16} \int_{Q}\left|z^{N}(x, t)\right|^{l^{\prime}} \mathrm{d} x+C_{17},
\end{aligned}
$$

其中 $2 \leqslant q l<l^{\prime}<\frac{2 m}{m-2}$ 且 $q>1$. 因此 $\left\{f\left(x, t, Z^{N_{i}^{\prime}}(x, t)\right)\right\}$ 当 $N_{i}^{\prime} \rightarrow \infty$ 时在 $L_{\infty}\left(0, T_{i q}^{\prime}\right.$ 
$\left.L_{1}(Q)\right)$ 所䎄收玫于 $f(x, t, z(x, t)$,$) .$

取极限过程 $N_{i}^{\prime} \rightarrow \infty$, 我们看到积分关系式(19)题于积分关系式(18). 因此 $n$ 维极限矢 量函数 $z(x, t)$ 是对铁滋链型旋方程组 $(4)(\varepsilon>0)$ 和方程组 $(1)(\varepsilon=0)$ 的齐次边值问题

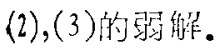

定理 1 假设条件 (I), (II) 和（III）满足. 对铁磁链型齐次旋方程组 (4) $(\varepsilon>0)$ 或 方程组 (1) $(\varepsilon=0)$ 的齐次边值问题 (2)，(3)侟至少一个弱解 $z(\boldsymbol{x}, \boldsymbol{t}) \in L_{\infty}\left(0, T ; H_{\mathrm{J}}(\Omega)\right) \cap$ $\left.C^{\left(0,-\frac{1}{2-1}\left[\frac{m}{2}\right]\right.}\right)\left([0, T] ; L_{2}(\Omega)\right)$.

定理 2 假设条件 (I), (II) 和 (III) 满足. 对齐次旋方型组 (4)的齐次边值问题 (2), (3)的弱解的任一收敛序列 $\left\{z^{z}(x, t)\right\}$, 当 $\varepsilon \rightarrow 0$ 的在

$$
L_{\infty}\left(0, T ; H_{v}^{1}(\Omega)\right) \cap \mathrm{C}^{\left(0, \frac{1}{2+\left[\frac{m}{2}\right]}\right)}\left([0, T] ; L_{2}(\Omega)\right)
$$

中的 $n$ 维极限矢量函数 $z(x, t)$ 是对位的铁磁链型方程组 (1) 的齐次边值问题 $(2)$, (3) 的弱 解。

证 $\forall \varepsilon>0$, 至少存一个 $n$ 维次量函数 $z^{\varepsilon}(x, t) \in G$ 茯足积分关系式

$$
\begin{aligned}
& \iint_{Q_{T}}\left\{\psi_{t}(x, t) z^{\varepsilon}(x, t)-\nabla \phi(x, t) \circ^{*}\left[z^{\varepsilon}(x, t) \wedge a_{2} \wedge \cdots \wedge a_{n-2} \wedge \nabla z^{\varepsilon}(x, t)\right]\right. \\
& \left.\quad-\varepsilon \nabla \psi(x, t) \circ \nabla z^{\varepsilon}(x, t)+\psi(x, t) f\left(x, t, z^{\varepsilon}(x, t)\right)\right\} \mathrm{d} x \mathrm{~d} t \\
& \quad+\int_{\Omega} \psi(x, 0) \varphi(x) \mathrm{d} x=0,
\end{aligned}
$$

其中 $\phi(x, t)$ 退佂一连续可微试验函数。

因为当 $\varepsilon \rightarrow 0$ 付

$$
\varepsilon \iint_{\dot{Q}} \nabla \psi(x, t) \nabla \nabla z^{\varepsilon}(x, t) \mathrm{d} x \mathrm{~d} t \rightarrow 0 .
$$

于是 $n$ 维枚限尔量函数 $z(x, t) \in G$ 满足积分关系式

$$
\begin{gathered}
\iint_{0}\left\{\psi_{t}(x, t) z(x, t)-\nabla \psi(x, t) 0^{*}\left[z(x, t) \wedge a_{2} \wedge \cdots \wedge a_{x-2} \wedge \nabla z(x, t)\right]\right. \\
+\phi(x, t) f(x, t, z(x, t))\} \mathrm{d} x \mathrm{~d} t+\int_{0} \psi(x, 0) \varphi(x) \mathrm{d} x=0 .
\end{gathered}
$$

因此 $z(x, t) \in L_{\infty}\left(0, T ; H_{0}^{1}(\Omega)\right) \cap \mathrm{C}^{\left(0, \frac{1}{2+\left[\frac{m}{2}\right]}\right)}\left([0, T] ; L_{2}(Q)\right)$ 是对铁磁链型齐次方程组

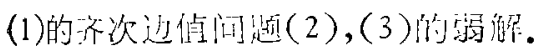

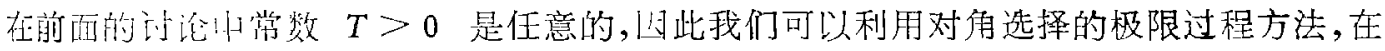
无穷长柱形域 $Q_{\infty}=\{x \in \bar{\Omega} ; 0 \leqslant t<\infty\}$ 中, 对上面所提到的问题构造弱的。这样我们有下 列对 $T=\infty$ 情形们题盟。

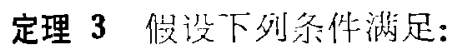

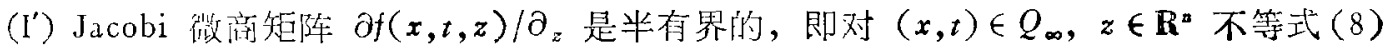
对任一秋量 $\xi \in \mathbb{R}^{n}$ 娍站; 
(II') 方程组 (1) 和(4)都是齐次的, 即 $f(x, t, 0) \equiv 0$. 对任一 $0<T<\infty$ 和 $(x, t) \in Q_{t}$, $\boldsymbol{z} \in \mathbb{R}^{n}$ 有

$$
\begin{aligned}
& |f(x, t, z)| \leqslant A(T)|z|^{l}+B(T), \\
& |\nabla f(x, t, z)| \leqslant A(T)|z|^{1+\frac{2}{m}}+B(T),
\end{aligned}
$$

其㳈 $A(T)$ 和 $B(T)$ 阵续依赖于 $T$ 和 $2<l<\frac{2 m}{m-2}, m \geqslant 2$;

(III) $n$ 维矢昆涵数 $\varphi(x) \in H_{0}^{\prime}(\Omega)$.

于是, 对铁逆链型方方程组 $(4)(\varepsilon>0)$ 或方程组 $(1)(\varepsilon=0)$ 的齐次边值问题 (2), (3)在元 穷柱形域 $Q_{\infty}$ 中至少有一个 $n$ 维红量解

$$
z(x, t) \in L_{\infty, h ; c}\left(\mathbb{R}^{+} ; H_{0}^{1}(\Omega)\right) \cap \mathrm{C}_{\text {loc }}^{\left(0, \frac{1}{2+\left[\frac{m}{2}\right]}\right)}\left(\mathbb{R}^{+} ; L_{2}(\Omega)\right) .
$$

在常数 $b<0$ 的情形, 当方程组 (4)和(1) 是齐次的, 即 $f(x, t, 0) \equiv 0$, 从 Galerkin 近储 解 $z^{N}(x, t)$ 所满足的不等式

$$
-\frac{\mathrm{d}}{\mathrm{d} t}\left\|z^{N}(\cdot, t)\right\|\left\|_{L_{2}(\Omega)}^{2} \leqslant-2|b|\right\| z^{N}(\cdot, t) \|_{L_{2}(\Omega)}^{2}
$$

知

$$
\left\|z^{N}(\cdot, t)\right\|_{L_{2}(\Omega)} \leqslant \|\left(p \|_{L_{1}(\Omega) e^{-|b| t}}, 0 \leqslant t<\infty .\right.
$$

因此, 当 $N \rightarrow \infty$ 肘对极限函数 $z(x, t)$ 的类似不等式成立。

定理 4 假设条件 $\left(\mathrm{I}^{\prime}\right),\left(\mathrm{I}^{\prime}\right)$ 和 (III) 满足且 $b<0$. 于是, 通过极限过程听得到的、 对珗磁链型旋方程组 (4) $(\varepsilon>0)$ 或方程组 $(1)(\varepsilon=0)$ 的齐次边值问题 (2), (3) 的任一果 解 $\approx(x, t)$, 有

$$
\lim _{z \rightarrow \infty}\|z(\cdot, t)\|_{\boldsymbol{L},(\Omega)}=0
$$

\section{参考文 触}

[1] Zhou Yulin, Lesture Norcs in Num. Afpl. Anal, 1982, 5: 435-457.

[2] Zhou Yulin, Sun Hesheng, Guo Boling. Preprint IfPCM 92-07, June 1992.

[3] Zhou Yulin, Sun Hesheng. Guo Boling, Aduance in Mazh., 1992, 21(4); 497-501.

[4] Zhou Yulin, Sun Hesheng. Guo Buline, Procicdings of the "International Conference on Nonlinear PDE, May 30June 3, langzhon, China, 1992

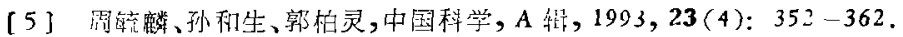

[6] Nakamura, K. Sasada, T., Phy. Letr., 1974, 48 A: i21-322.

[7] Tjon, J., Wright, J., Phy. Reu., 1977, 15B: $3470-3476$.

[8] Fogedby, H. C., Lecture Notes in Physics, Vol. 131, Springer-Verl.ng, Berlin, 1980.

[9] Takhtajin, L. A., Phy Lett., 1977, 64 A: 2j3.

[10] Zhou Yulin, Guo Boling, Prac. of DL-3 Symposium, 1982,713-732.

[11] Zhou Yulin, Guo Boling, Scientia Sinica, Ser. A, 1984, 27: 799-811.

[12] Zhou Yulin, Guo Boling, Scienita Sinica, Ser. A, 1987, 30: 1251-1266.

[13] Zbou Yulin, Guo Bolıng, Tan Shuobin, Science in China, Ser. A, 1991, 34(3): 257-266. 